\title{
Oficina culinária como estratégia de articulação entre os movimentos sociais e a comunidade acadêmica para a promoção da alimentação saudável e sustentável: relato de experiência
}

\author{
Bruna Carraco de Azeredo Peres ${ }^{1}$, Marianna Miranda Rodrigues Vidal ${ }^{1}$, Larissa Paulino Gama ${ }^{1}$, Érica Ribeiro \\ Pires $^{1}$, Desirée Lopes Reis ${ }^{2}$, Marcio Marques Silva33, Mara Lima De Cnop ${ }^{3}$, Avany Fernandes Pereira3 ${ }^{3}$ Thadia \\ Turon Costa da Silva ${ }^{4}$
}

Resumo: A alimentação da população brasileira apresenta o baixo consumo de frutas e hortaliças, pouca variedade de espécies alimentícias, além do elevado consumo de alimentos ultraprocessados, o que contribui para o desenvolvimento de doenças crônicas não transmissíveis. O desenvolvimento das habilidades culinárias resgata a cultura alimentar e promove a alimentação saudável e sustentável. Este trabalho relata a experiência de uma oficina culinária com a hortaliça chaya (Cnidoscolus aconitifolius) como principal ingrediente, realizada na V Jornada Universitária em Defesa da Reforma Agrária na UFRJ (2018) em parceria com o Movimento dos Trabalhadores Rurais Sem Terra (MST). As etapas da ação foram planejamento da oficina, pesquisa e elaboração dos materiais de apoio e didático, execução da oficina e degustação das preparações, por meio de um teste de aceitabilidade. A oficina iniciou com uma roda de conversa para apresentar orientações sobre a ação e possibilitar diálogos, seguida pela execução das preparações culinárias e degustação. Participaram da atividade 12 pessoas, com idade entre 19 e 70 anos, e $67 \%$ eram mulheres. Na roda de conversa, observou-se grande interesse e troca de saberes entre os participantes, o que demonstra que as metodologias utilizadas se adequaram ao público participante. As preparações elaboradas na oficina culinária foram: massa fresca de chaya com molho de tomate, torta de ricota com chaya, arroz de cuxá, grissini de chaya e bolo com chaya. As preparações tiveram Índice de Aceitabilidade (IA) maior que $85 \%$ para todos os atributos sensoriais avaliados, verificando-se interesse em reproduzi-las casa. Concluiu-se que as oficinas culinárias podem articular os movimentos sociais e a comunidade acadêmica, fomentando a alimentação saudável.

Palavras-chave: Extensão Universitária; Agrobiodiversidade; Cnidoscolus aconitifolius; Habilidades Culinárias

\section{Culinary workshop as an articulation strategy between social movements and the academic community to promote healthy and sustainable diets: an experience report}

Abstract: The eating habits of the Brazilian population are characterized by low consumption of fruits and vegetables, little variety of edible species, besides the high consumption of ultra-processed food, which contributes to non-transmissible chronic diseases. The development of cooking skills promotes rediscovering food culture and a healthy and sustainable diet. The objective of this paper was to report the experience of a culinary workshop using chaya (Cnidoscolus aconitifolius) as the main ingredient held during the 5th University Journey for the Support of Agrarian Reform at UFRJ (2018) in partnership with the Landless Rural Workers Movement (MST). The action had the stages of planning the culinary workshop, researching and preparing the support and didactic material, holding the workshop, and sensory evaluation of the preparations, using an acceptability test. The workshop began with a round-table discussion initially held to provide guidelines about the action and allow dialogues, followed by the culinary preparations and tasting. Twelve people between the ages of 19 and 70 participated in the activity, $67 \%$ of whom were women. During the round-table discussion, great interest and knowledge exchange among all participants were observed, showing that the methodologies were adequate to the participants. The preparations developed during the cooking workshop were: fresh chaya pasta with tomato sauce, ricotta pie with chaya, cuxá rice, chaya grissini, and (E) cake with chaya. The preparations made during the workshop presented an Acceptability Index (AI) higher than $85 \%$ for all evaluated sensory attributes. The participants also showed an interest in reproducing the workshop preparations at home. It was concluded that culinary workshops could be essential to articulate social movements and the academic community and promote a healthy diet.

Keywords: University Extension; Agrobiodiversity; Cnidoscolus aconitifolius; Culinary Skills

Originais recebidos em

21 de julho de 2020

Aceito para publicação em

15 de abril de 2021

1

Graduanda de Nutrição da Universidade Federal do Rio de Janeiro (UFRJ) e bolsista de extensão PR5

\section{2}

Doutoranda do Programa de Pós Graduação em Nutrição da UFRJ.

3 Docente do Instituto de Nutrição Josué de Castro da Universidade Federal do Rio de Janeiro (UFRJ)

4

Docente do Instituto de Nutrição Josué de Castro da Universidade Federal do Rio de Janeiro (UFRJ)

(autora para correspondência) thadiaturon@nutricao.ufrj.br 


\section{Introdução}

Em mais de $90 \%$ da população brasileira, o consumo diário de frutas e hortaliças está abaixo da quantidade mínima recomendada para prevenção das doenças crônicas não transmissíveis, o que corresponde a 400g por dia (Ministério da Saúde, 2011; Jaacks et al., 2019). Apesar de incluir quase dois terços de alimentos in natura ou minimamente processados, o consumo atual do brasileiro apresenta pouca variedade, totalizando um registro de apenas 26 espécies vegetais, embora estime-se que, no Brasil, existam cerca de 10 mil espécies vegetais com potencial alimentício (Instituto Brasileiro De Geografia e Estatística [IBGE], 2011; Kinupp \& Lorenzi, 2014). Ao mesmo tempo, há um aumento no consumo diário de produtos ultraprocessados, alcançando em torno de $25,4 \%$ da alimentação do brasileiro, em substituição aos alimentos como frutas, laticínios, assim como das preparações culinárias nas refeições principais (Martins et al., 2013; Ministério da Saúde, 2014).

O aumento da contribuição energética da dieta proveniente de alimentos ultraprocessados está diretamente associado ao consumo de nutrientes como açúcares de adição, gordura saturada, e inversamente associada a consumo proteico e de fibras (Marrón-Ponce et al., 2019). Este fato pode contribuir para maior risco de desenvolver doenças crônicas tais como obesidade, diabetes mellitus, hipertensão arterial sistêmica, doença arterial coronariana, e alguns tipos de neoplasias (Ministério da Saúde, 2017).

O Guia Alimentar para a população brasileira define produtos ultraprocessados como

"(...) formulações industriais feitas inteiramente ou majoritariamente de substâncias extraídas de alimentos (óleos, gorduras, açúcar, amido, proteínas), derivadas de constituintes de alimentos (gorduras hidrogenadas, amido modificado) ou sintetizadas em laboratório com base em matérias orgânicas como petróleo e carvão (corantes, aromatizantes, realçadores de sabor e vários tipos de aditivos usados para dotar os produtos de propriedades sensoriais atraentes)." (Ministério da Saúde, 2014, p. 41).

Além dos fatores motivadores relacionados à saúde, a orientação de se evitar o consumo de produtos ultraprocessados decorre de sua intrínseca relação com um modelo de agronegócios voltado ao uso abusivo e frequente de agrotóxicos, monoculturas de milho, soja, trigo e cana-de-açúcar, além da gestão precária de resíduos, impactando diretamente na vida social e no meio ambiente (Ribeiro et al., 2017).

O desenvolvimento das habilidades culinárias parece ter grande impacto no resgate da cultura alimentar e na promoção da alimentação saudável, sustentável e diversificada, visto que informações sobre técnicas dietéticas podem melhorar a qualidade da informação sobre o consumo e as práticas alimentares e, assim, estimular mudanças alimentares, pelo fato de abordar suas dimensões sensoriais, cognitivas, simbólicas e práticas (DiezGarcia \& Castro, 2011).

As práticas alimentares promotoras de saúde devem respeitar a diversidade cultural e as inserções sócioeconômicas e ambientais de forma sustentável (Castro et al., 2007). Neste sentido, o projeto de extensão universitária "Biodiversidade, cultura alimentar e gastronomia: degustando novos saberes" vinculado ao Instituto de Nutrição Josué de Castro (INJC) da Universidade Federal do Rio de Janeiro (UFRJ), realiza atividades de educação alimentar e oficinas culinárias, desenvolvendo receitas com uso predominante das frutas e hortaliças não convencionais, no intuito de ampliar a utilização cotidiana desses alimentos, promovendo o cultivo e sua comercialização, e fortalecendo esta tradição junto à rede de consumidores e agricultores familiares e agroecológicos do Rio de Janeiro. O presente trabalho teve como objetivo relatar a experiência de uma oficina culinária realizada durante $V$ Jornada Universitária em Defesa da Reforma Agrária realizada na UFRJ em 2018, em parceria com o Movimento dos Trabalhadores Rurais Sem Terra (MST), possibilitando a 
articulação entre os movimentos sociais e a comunidade acadêmica para discussão de temas relacionados à agricultura e produção de alimentos, biodiversidade alimentar, alimentação saudável e sustentável, trabalho no campo e a luta contra o uso de agrotóxicos.

\section{Metodologia}

A oficina culinária foi planejada seguindo a proposta pedagógica idealizada por Freire (1977), constituindo-se como uma prática crítico-educativa, privilegiando o diálogo, o respeito pelos saberes do educando, e o reconhecimento da identidade cultural, levando em consideração a apropriação do conhecimento por ambas as partes.

A ação contou com as etapas de planejamento da oficina culinária, pesquisa e elaboração do material de apoio e didático, execução da oficina e avaliação. A equipe de orientadores da oficina foi composta por dois professores do curso de graduação em Nutrição e dois do curso de Gastronomia. A equipe extensionista foi composta por quatro alunos de graduação em Nutrição e um em Gastronomia, e um pós-graduando em Ciências dos Alimentos da UFRJ. Toda equipe participante era integrante do projeto.

A oficina foi aberta à comunidade participante da V Jornada Universitária em Defesa da Reforma Agrária realizada na UFRJ, composta em sua maioria por discentes e docentes da universidade, líderes e coordenadores de acampamentos e assentamentos do MST, e foi realizada em parceria com o coletivo "Empório da chaya", um grupo de mulheres do acampamento Marly Pereira da Silva, Paracambi, RJ (MST), que tem a chaya como principal hortaliça cultivada, usada nas preparações culinárias elaboradas por esse coletivo e como forma de empoderamento e resistência.

$\mathrm{Na}$ etapa de planejamento, foram definidos os temas disparadores para uma roda de conversa inicial, as receitas que seriam executadas e o conteúdo a ser abordado nos materiais didáticos. Para a definição das preparações culinárias foi realizada busca na literatura científica sobre a chaya (Cnidoscolus aconitifolius), teste no laboratório culinário das receitas propostas, e elaboração das fichas técnicas de preparo. Além disso, foram desenvolvidas as apostilas com as receitas, o questionário de avaliação sensorial das preparações culinárias, e o formulário de avaliação da oficina. A roda de conversa foi utilizada como ferramenta metodológica inicial para comunicação dinâmica e produtiva entre os participantes (Sampaio et al., 2014).

Após a execução das receitas, os participantes degustaram as preparações culinárias e avaliaram cada preparação em relação ao aspecto, consistência, aroma e sabor, por meio de formulário contendo escala hedônica de 5 pontos, com respostas variando do adorei (5 pontos) ao detestei (1 ponto) (Meilgaard et. al., 1999). As notas foram expressas com a média \pm desvio padrão, e foi considerada boa aceitabilidade quando $o$ Índice de Aceitabilidade (IA) foi maior que 70\%. O IA (\%) foi calculado tendo como base as notas obtidas no teste de aceitabilidade para cada preparação por meio da fórmula: $A$ x 100/B, na qual $A$ corresponde à nota média obtida para a preparação e $B$, a nota máxima dada à preparação (Dutcosky, 2013). Na ficha de avaliação também constavam informações sociodemográficas dos participantes, entre outras (Material Suplementar).

\section{Resultados}

\section{Caracterização da chaya}

Muitas frutas, hortaliças e outras plantas comestíveis, nativas ou exóticas, espontâneas ou cultivadas, não estão incluídas no cardápio cotidiano da população, e são também denominadas "não-convencionais". As espécies que se enquadram nesse conceito são aquelas que ainda não receberam a devida atenção por parte da 
comunidade técnico-científica e da sociedade como um todo, resultando em seu consumo localizado e pontual em algumas regiões, exercendo grande influência na alimentação e na cultura de populações tradicionais, e com dificuldade de penetração nas demais populações do país. Este fato está relacionado por serem cultivos que não estão organizados enquanto cadeia produtiva propriamente dita, diferentemente das hortaliças convencionais (por exemplo, batata, tomate, repolho, alface), por não terem demanda de mercado, além de não despertarem interesse por parte de empresas de agronegócio, tais como de fertilizantes, agroquímicos ou indústria de alimentos (Ministério da Agricultura, Pecuária e Abastecimento, 2010).

Estas hortifrutícolas, hoje marginalizadas, expressam a biodiversidade brasileira e são fontes subexploradas de nutrientes e compostos bioativos que oferecem efeitos positivos à saúde, além de sabores e aromas desconhecidos pela população (Kelen et al., 2015; Biazotto et al., 2019), que precisam ser exploradas e ampliadas quanto ao uso, resgatando conhecimento sobre formas de cultivo, manejo, sazonalidade, conservação pós-colheita e uso destas na culinária.

A chaya (Cnidoscolus aconitifolius), considerada uma hortaliça não convencional, é uma espécie nativa do México, cultivada em escala doméstica na América Central e América do Sul, para produção de folhas comestíveis, de coloração verde escura similar à couve. É um arbusto perene de crescimento rápido, que chega a possuir até seis metros de altura. Seu cultivo é geralmente feito com estacas de caule, sendo melhor em ambiente ensolarado, água em abundância, temperatura média de $25^{\circ} \mathrm{C}$ e altitude variando entre 0 e 1.000 metros acima do nível do mar. No entanto, apresenta-se pouco exigente e bastante resistente em condições edafoclimáticas adversas (Munguía-Rosas et al., 2019).

É recomendado o consumo desta hortaliça cozida, refogada ou fervida, a fim de hidrolisar os glicosídeos cianogênicos tóxicos presentes nela (Jiménez-Aguilar \& Grusak, 2015). Nos lugares em que seu consumo é tradicional, as folhas de chaya são geralmente usadas em chás, sopas, tamales, ou cozidas com outros vegetais, legumes, carne ou ovos (Kuti \& Kuti, 1999).

Quando comparada a outras hortaliças (como por exemplo o espinafre, couve-manteiga e rúcula) possui igual ou maior teor de proteínas, cálcio, ferro, fibras, vitamina A e vitamina C (Kuty \& Torres, 1996; Ross-Ibarra \& Molina-Cruz, 2002). A literatura científica destaca, entre as propriedades da folha, atividade hipoglicemiante, anti-inflamatória, antiprotozoária, antimicrobiana e antioxidante (Soto et al, 2015; García-Rodríguez \& Gutiérrez-Rebolledo, 2018).

Para o coletivo Empório da chaya, essa hortaliça é considerada símbolo de resistência, por mostrar-se tolerante a condições ambientais adversas e solos inférteis para a agricultura, muito comum nas terras convertidas em assentamento da reforma agrária, de difícil recuperação e produção de outros alimentos.

\section{Relato de Experiência}

A oficina foi realizada em abril de 2018 no Laboratório de Técnica Dietética do Restaurante Universitário Central da Universidade Federal do Rio de Janeiro (UFRJ), com duração de 5 horas. Participaram da atividade 12 pessoas com idade entre 19 a 70 anos. Do total de participantes, $67 \%$ eram mulheres, $42 \%$ possuíam ensino médio completo, e $25 \%$ ensino fundamental completo ou incompleto (Tabela 1 ).

A oficina iniciou-se com uma roda de conversa, seguida de orientações sobre segurança e higiene no laboratório, apresentação, execução e avaliação das receitas (Figura 1). Após a apresentação das receitas, seguiu-se a discussão, utilizando como temas disparadores: conhecimento e consumo de hortaliças não convencionais, biodiversidade e alimentação saudável. A roda de conversa, com duração de cerca de quarenta minutos, foi realizada previamente à elaboração das preparações, como ferramenta metodológica para comunicação dinâmica e produtiva entre os participantes. As discussões foram pautadas nas percepções dos 
participantes sobre a relação da produção, comercialização e escolha dos alimentos, e sua influência nas relações humanas e ambientais. A partir dos temas disparadores utilizados, foi oportunizado a todos os presentes testemunharem um relato emocionante de uma das participantes sobre suas experiências e vivências de nove anos em um acampamento do MST, às margens de uma rodovia, onde teve a oportunidade de cultivar a chaya.

Ao final, os 12 participantes degustaram as preparações executadas e procederam a avaliação das preparações em relação ao aspecto, consistência, aroma e sabor. Conforme apresentado na Tabela 2, os participantes adoraram e/ou gostaram de todos os quesitos avaliados do grissini, da torta de ricota e do bolo. Todos os participantes relataram que adoraram e/ou gostaram do aspecto e sabor da massa fresca, e 92\% apresentaram o mesmo relato para a consistência e aroma. Cerca de $92 \%$ dos participantes disseram que adoraram/gostaram de todos os quesitos do arroz de cuxá. Todas as preparações apresentaram Índice de Aceitabilidade maior que 85\% para todos os atributos (Tabela 3). A sabor, cor e aspectos da chaya nas preparações culinárias lembram o espinafre o que pode explicar os altos IA obtidos (Porres e Cifuentes 2014). Apesar de pouco conhecida no Brasil, as folhas da chaya são largamente utilizadas no México em preparações culinárias para melhorar a composição nutricional e a aceitação sensorial dos alimentos (Guillermo-Moreno, et al. 2019).

Tabela 1. Caracterização do perfil dos participantes da oficina culinária do projeto "Biodiversidade, cultura alimentar e gastronomia: degustando novos saberes", abril de 2018 .

\begin{tabular}{cc}
\hline Faixa Etária & $\%$ \\
\hline $18-37$ & 41,6 \\
$38-57$ & 50 \\
$58-78$ & 8,3 \\
\hline Sexo & $\%$ \\
\hline Masculino & 33,3 \\
\hline Feminino & 66,70 \\
\hline Estado Civil & $\%$ \\
\hline Solteiro/a & 75,0 \\
\hline Casado/morando junto & 25,0 \\
\hline Viúvo/a & 00,0 \\
\hline Escolaridade & $\%$ \\
\hline Ensino Fundamental Incompleto & 8,3 \\
Ensino Fundamental Completo & 16,7 \\
Ensino Médio Completo & 41,7 \\
Ensino Superior Completo & 16,7 \\
Pós Graduação (mestrado, doutorado, especialização) & 8,3 \\
\hline
\end{tabular}



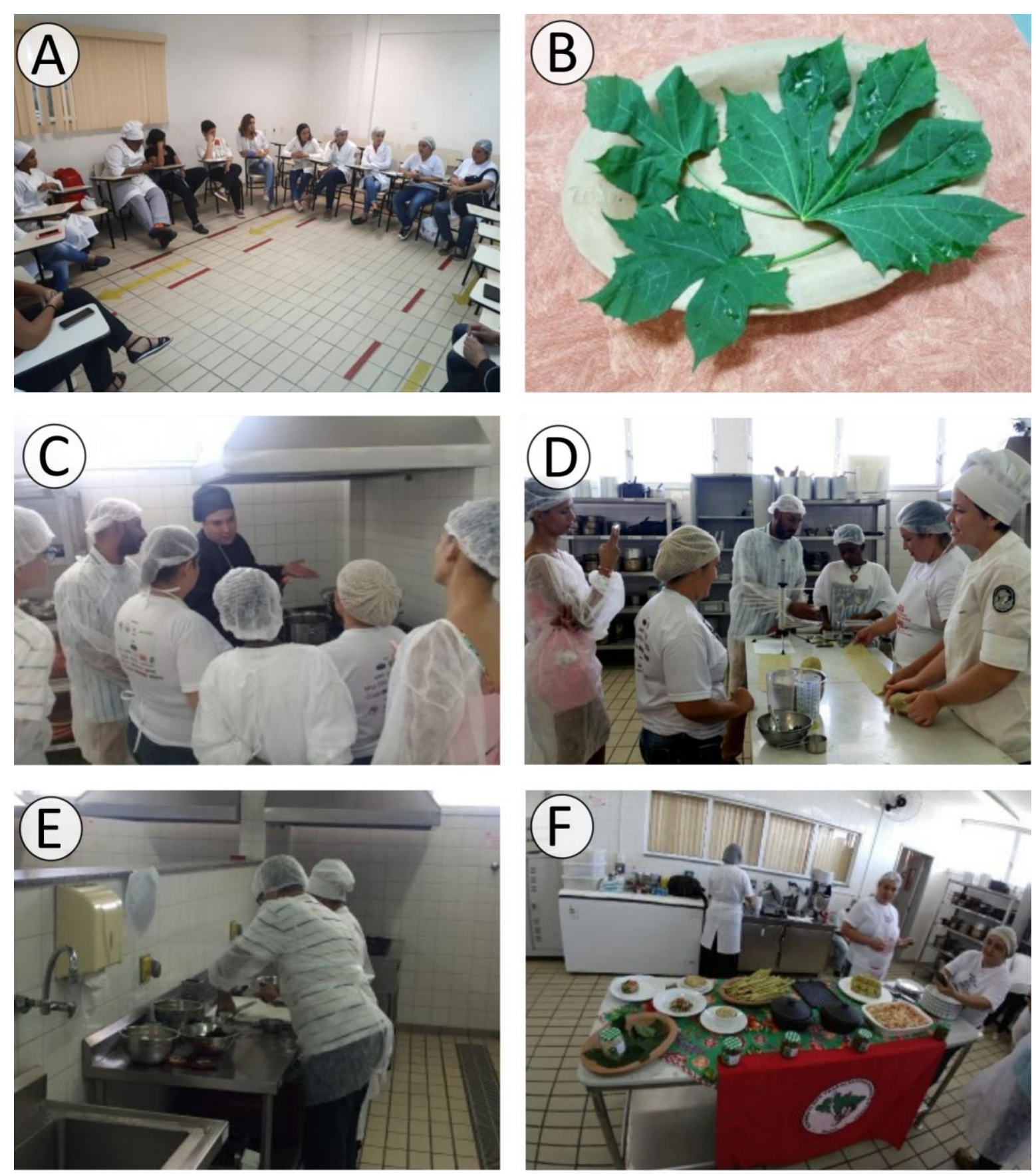

Figura 1. Etapas da oficina culinária desenvolvida na UFRJ, como parte do projeto "Biodiversidade, cultura alimentar e gastronomia: degustando novos saberes", abril de 2018. (A) roda de conversa, (B) apresentação da chaya, (C) apresentação das receitas, (D) e (E) execução das preparações, (F) mesa para degustação das preparações.

$\mathrm{Na}$ etapa seguinte, os participantes foram divididos em grupos e conduzidos às bancadas do laboratório culinário para a execução das receitas, junto aos integrantes da equipe. Foram executadas cinco preparações que utilizaram a hortaliça chaya e outros ingredientes culinários ou alimentos in natura como ingredientes: massa fresca, torta de ricota, arroz de cuxá, grissini e bolo (Figura 2). Não foram utilizados nas preparações produtos ultraprocessados, açúcar refinado, e soja, provenientes da agricultura agroquímica. 
Tabela 2. Notas médias e desvio padrão dos atributos sensoriais das preparações.

\begin{tabular}{lcccc}
\hline Preparações & Aspecto & Consistência & Aroma & Sabor \\
\hline Massa fresca de chaya & $4,92 \pm 0,29$ & $4,58 \pm 0,67$ & $4,42 \pm 0,67$ & $4,25 \pm 0,45$ \\
Grissini de chaya & $4,75 \pm 0,45$ & $4,83 \pm 0,39$ & $4,50 \pm 0,52$ & $4,67 \pm 0,49$ \\
\hline Torta de chaya & $4,92 \pm 0,29$ & $4,83 \pm 0,39$ & $4,83 \pm 0,39$ & $4,92 \pm 0,29$ \\
Arroz de Cuxá & $4,75 \pm 0,62$ & $4,58 \pm 0,67$ & $4,67 \pm 0,65$ & $4,83 \pm 0,58$ \\
\hline Bolo de chaya & $4,83 \pm 0,39$ & $4,83 \pm 0,39$ & $4,83 \pm 0,39$ & $4,83 \pm 0,39$ \\
\hline
\end{tabular}
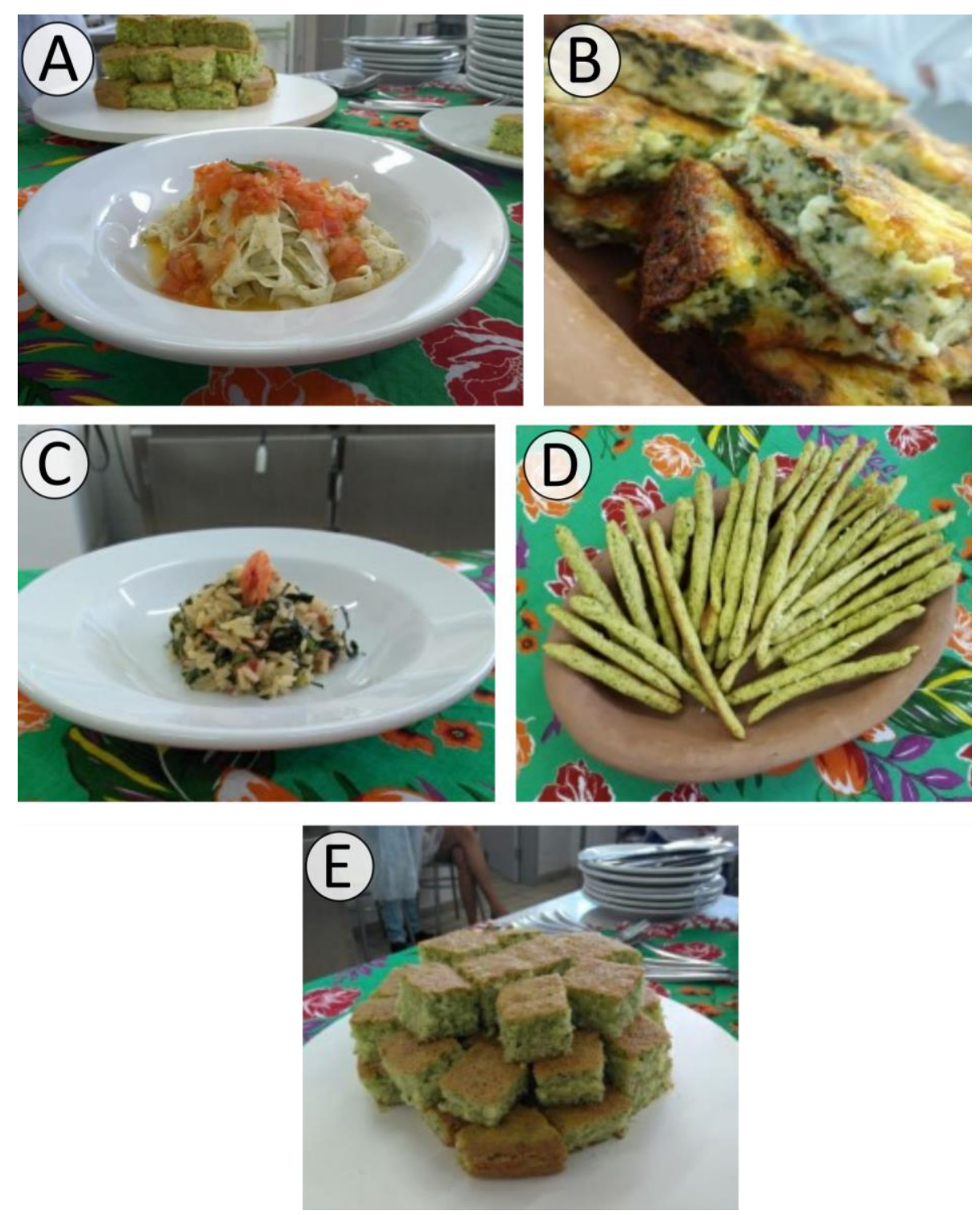

Figura 2. Preparações elaboradas na oficina culinária (A) massa fresca de chaya com molho de tomate, (B) torta de ricota com chaya, (C) arroz de cuxá, (D) grissini e (E) bolo. 
Tabela 3. Valores médios do Índice de Aceitabilidade (IA) das preparações por atributos das preparações, valores em porcentagem.

\begin{tabular}{lcccc}
\hline Preparações & Aspecto & Consistência & Aroma & Sabor \\
\hline Massa fresca com chaya & 98,33 & 91,67 & 88,33 & 85,00 \\
Grissini de chaya & 95,00 & 96,67 & 90,00 & 93,33 \\
\hline Torta de chaya & 98,33 & 96,67 & 96,67 & 98,33 \\
Arroz de Cuxá & 95,00 & 91,67 & 93,33 & 96,67 \\
Bolo de chaya & 96,67 & 96,67 & 96,67 & 96,67 \\
\hline
\end{tabular}

Metade dos participantes relatou que já conheciam a chaya e todos disseram que comprariam essa hortaliça não convencional. Em relação ao potencial de compra da massa fresca e do grissini, $92 \%$ e $75 \%$ dos participantes, respectivamente, relataram que certamente comprariam essas preparações caso fossem comercializadas. Dos participantes, $92 \%, 83 \%$ e $75 \%$, relataram que fariam em casa a torta de ricota, o arroz de cuxá e o bolo, respectivamente. Esse resultado evidencia a aceitação por parte dos participantes à diversificação da alimentação, e o interesse em consumir hortaliças não convencionais.

\section{Discussão}

As rodas de conversas possibilitam encontros dialógicos, criando possibilidades de produção e ressignificação de sentido sobre as experiências dos partícipes. Na presente ação, essa técnica apresentou-se como rico instrumento para ser utilizado como meio de aproximação entre os sujeitos no cotidiano pedagógico. Como metodologia de trabalho com coletivos, a roda de conversa vem sendo desenvolvida em diversos contextos, a partir dos estudos Freirianos. Freire (1996) aponta que exercitaremos tanto mais e melhor a nossa capacidade de aprender e de ensinar, quanto mais sujeitos e não puros objetos do processo nos façamos. Nesse sentido, a roda de conversa, para além da disposição física circular dos indivíduos, proporciona postura ética-política em relação à produção do conhecimento e à transformação social, efetivando-se a partir das negociações entre sujeitos (Sampaio et al., 2014).

A falta de habilidade culinária e a menor frequência de preparo domiciliar dos alimentos podem ser uma barreira à realização da alimentação saudável, pois predispõe os indivíduos a consumirem alimentos prontos para o consumo, que estão associados com baixa qualidade da dieta, sobrepeso e obesidade (Van Der Horst et al., 2011; Laska et al., 2012; Reicks et al., 2014;). O Guia Alimentar para a População Brasileira destaca a necessidade de retomar as práticas culinárias como forma de redução de consumo de alimentos processados e ultraprocessados, uma vez que, no ato de cozinhar se utiliza, majoritariamente, alimentos in natura e minimamente processados. Além disso promove-se a manutenção e propagação das habilidades culinárias para as novas gerações.

Para Castro et al. (2007), a oficina culinária funciona como um cenário capaz de sensibilizar e incentivar mudanças em relação às práticas e habilidades culinárias e sua relação com a saúde, a sociedade e o ambiente. Além disso, pode-se considerar que a culinária é uma prática social que conecta elementos individuais e coletivos, agrega o conhecimento tradicional e também informações advindas de outras vivências. A culinária se apresenta como espaço apropriado e promissor para intervenções que visem promover a troca de 
experiências e aprendizado holístico sobre alimentação (Castro et al., 2007). Assim, iniciativas como essas corroboram com as diretrizes de segurança alimentar e nutricional propostas no país (Ministério da Saúde, 2014).

Os resultados apresentados neste trabalho demonstram que a as preparações culinárias tiveram uma boa aceitação (IA > 85\% para todos os atributos), além do interesse em reproduzir as preparações culinárias da oficina em casa. Isso demonstra que ações de extensão que promovem a troca de saberes sobre plantas alimentícias não-convencionais podem ser uma estratégia para somar o conhecimento acerca de uma planta nutritiva, porém pouco consumida. Além disso, esse tipo de metodologia pode estimular os participantes a ampliar as habilidades culinárias, com o intuito de fortalecer o que é abordado no Guia Alimentar para a População Brasileira.

\section{Considerações finais}

A oficina culinária, como prática educativa, configurou-se pertinente para a promoção da alimentação saudável e sustentável. Aliada à roda de conversa, tal proposta viabilizou a relação dos alunos e professores componentes da equipe do projeto com agricultores membros do MST em uma ação marcada pelo diálogo, ação de mão-dupla e troca de saberes. A partir da ação relatada, foi possível observar o cumprimento das diretrizes da extensão universitária por meio da troca de conhecimentos, da participação e do contato com as questões complexas contemporâneas em seu contexto social, além da contribuição na formação integral do estudante extensionista, estimulando sua formação como cidadão crítico e responsável.

Foi observado um grande interesse dos participantes sobre o tema em tela, o que demostra que a metodologia utilizada contribuiu para o alcance dos objetivos. As preparações elaboradas na oficina gastronômica tiveram boa aceitação, e podem ser um estímulo para aumento da produção e consumo de alimentos in natura, principalmente hortaliças não convencionais, contribuindo para promoção da alimentação saudável, consciente e sustentável.

\section{Agradecimentos}

Agradecemos à Pró-Reitoria de Extensão da UFRJ (PR-5) pelo apoio financeiro que contribuiu para a realização da ação e das bolsas dos extensionistas.

\section{Contribuição de cada autor}

Todos os autores trabalharam em todas as etapas desde a concepção da ação de extensão relatada, execução, análise e interpretação dos dados. T.T.C.S, A.F.P, M.L.C e B.C.A.P participaram da redação do artigo e da sua versão final. T.T.C.S, A.F.P e M.L.C atuaram como coordenadoras e orientadoras dos bolsistas.

\section{Referências}

Biazotto, K. R., Mesquita, L. M. S., Neves, B. V., Braga, A. R. C., Tangerina, M. M. P., Vilegas, W., ... \& Rosso, V. V. (2019). Brazilian biodiversity fruits: Discovering bioactive compounds from underexplored sources. Journal of Agricultural and Food Chemistry, 67(7), 1860-1876.

Castro, I. R. R., Souza, T. S. N., Maldonado, L. A., Caniné, E. S., Rotenberg, S., \& Gugelmin, S. A. (2007). A culinária na promoção da alimentação saudável: Delineamento e experimentação de método educativo dirigido a adolescentes e a profissionais das redes de saúde e de educação. Revista de Nutrição, 20(6), 571-588. 
Diez-Garcia, R. W., \& Castro, I. R. R. (2011). A culinária como objeto de estudo e de intervenção no campo da Alimentação e Nutrição. Ciência \& Saúde Coletiva, 16(1), 91-98.

Dutcosky, S. D. (2013). Análise sensorial de alimentos. 4. ed. Curitiba: Champagnat.

Freire, P. (1977). A mensagem de Paulo Freire. Teoria e prática da libertação. 1. Ed. Porto: Nova Crítica.

Freire, P. (1996). Pedagogia da Autonomia: Saberes necessários à prática educativa. São Paulo: Paz e Terra.

García-Rodríguez, R.V., \& Gutiérrez-Rebolledo, G.A. (2013). Cnidoscolus chayamansa Mc Vaugh, an important antioxidant, anti-inflammatory and cardioprotective plant used in Mexico. Journal of Ethnopharmacology, 151(2), 937943.

Guillermo-Moreno, R.; Durán-Mendoza, T.; González-Cortés; N.; \& Jiménez-Vera, R. (2019) Calidad sensorial de totopos de pozol adicionados con chaya (Cnidoscolus aconitifolius) y hierba mora (Solanum nigrum). European Scientific Journal, 15(3), 15-27.

Instituto Brasileiro de Geografia e Estatística. (2011). Pesquisa de orçamentos familiares 2008-2009: Análise do consumo alimentar pessoal no Brasil. IBGE, Coordenação de Trabalho e Rendimento. Rio de Janeiro: IBGE.

Jaacks, L. M., Vandevijvere, S., Pan, A., McGowan, C. J., Wallace, C., Imamura, F., ... \& Ezzati, M. (2019). The obesity transition: Stages of the global epidemic. The Lancet: Diabetes \& Endocrinology, 7(3), 231-240.

Jiménez-Aguilar, D. M., \& Grusak, M. A. (2015). Evaluation of Minerals, phytochemical compounds and antioxidant activity of mexican, central american, and african green leafy vegetables. Plant Food for Human Nutrition, 70(4), 357364.

Kelen, M. E. B., Nouhuys, I. S. V., Kehl, L. C., Brack. P., \& Silva, D. B. (2015). Plantas alimentícias não convencionais (PANCs): Hortaliças espontâneas e nativas. 1. ed. Porto Alegre: UFRGS.

Kinupp, V.F.; Lorenzi, H. (2014). Plantas Alimentícias não convencionais (PANC) no Brasil. Guia de identificação, aspectos nutricionais e receitas ilustradas. São Paulo: Plantarum.

Kuti, J. O., \& Kuti, H. O. (1999). Proximate composition and mineral content of two edible species of Cnidoscolus (tree spinach). Plant Foods for Human Nutrition, 53(4), 275-283.

Laska, M. N., Larson, N. I., Neumark-Sztainer, D., \& Story, M. (2011) Does involvement in food preparation track from adolescence to young adulthood and is it associated with better dietary quality? Findings from a 10-year longitudinal study. Public Health Nutrition, 15(7), 1150-1158.

Marrón-Ponce, J. A., Flores, M., Cediel, G., Monteiro, C. A., \& Batis, C. (2019). Associations between consumption of ultra-processed foods and intake of nutrients related to chronic non-communicable diseases in Mexico. Journal of the Academy of Nutrition and Dietetics, 119(11), 1852-1865.

Martins, A. P., Levy, R. B., Claro, R. M., Moubarac, J. C., \& Monteiro, C. A. (2013). The obesity transition: Stages of the global epidemic. Revista de Saúde Pública, 47(4), 656-665.

Meilgaard, M. C., Civille, G., \& Carr, B. (2016). Sensory Evaluation Techniques. Boca Raton: CRC Press.

Ministério da Agricultura, Pecuária e Abastecimento. (2010). Manual de hortaliças não-convencionais.- Brasília: Secretaria de Desenvolvimento Agropecuário e Cooperativismo, MAPA/ACS. Recuperado de http://www.abcsem. com.br/docs/manual hortalicas web.pdf

Ministério da Saúde. (2011). Plano de ações estratégicas para o enfrentamento das doenças crônicas não transmissíveis (DCNT) no Brasil 2011-2022. Brasília: Secretaria de Vigilância em Saúde. Departamento de Análise de Situação de Saúde. Ministério da Saúde. (Série B. Textos Básicos de Saúde). Recuperado de https://bvsms.saude.gov.br/bvs/ publicacoes/plano acoes enfrent_dcnt 2011.pdf

Ministério da Saúde. (2014). Guia alimentar para a população brasileira. 2. ed. Brasília: Ministério da Saúde, Secretaria de atenção à saúde, departamento de atenção Básica. 
Ministério da Saúde (2017). Vigitel Brasil 2016: Vigilância de fatores de risco e proteção para doenças crônicas por inquérito telefônico: Estimativas sobre frequência e distribuição sociodemográfica de fatores de risco e proteção para doenças crônicas nas capitais dos 26 estados brasileiros e no Distrito Federal em 2016. Brasília: Ministério da Saúde, Secretaria de Vigilância em Saúde, Departamento de Vigilância de Doenças e Agravos não Transmissíveis e Promoção da Saúde. Recuperado de http://portalarquivos2.saude.gov.br/images/pdf/2018/marco/02/vigitel-brasil-2016.pdf

Munguía-Rosas, M., Jácome-Flores, M., Bello-Bedoy, R., Solís-Montero, V., \& Ochoa-Estrada, E. (2019). Morphological divergence between wild and cultivated chaya (Cnidoscolus Aconitifolius) (Mill.) I.M. Johnst. Genetic Resources and Crop Evolution, 66, 1389-1398.

Porres, V., \& Cifuentes, R. (2014). La chaya una planta muy nutritiva. Proyecto UVG-USDA-FFPr10. Centro de Estudios Agrícolas y Alimentarios, CEAA. Guatemala: Universidad del Valle de Guatemala.

Reicks, M., Trofholz, A. C., Stang, J. S., \& Laska, M. N. (2014). Impact of cooking and home food preparation interventions among adults: outcomes and implications for future programs. Journal of Nutrition Education and Behavior, 46(4), 259276.

Ribeiro, H., Jaime, P. C., \& Ventura, D. (2017). Alimentação e sustentabilidade. Estudos Avançados, 31(89), $185-198$.

Ross-Ibarra, J., \& Molina-Cruz, A. (2002). The ethnobotany of chaya (Cnidoscolus aconitifolius ssp. aconitifolius Breckon): A nutritious Maya vegetable. Economtc Botany, 56(4), 350-365.

Sampaio, J., Santos, G. C., Agostini, M., \& Salvador, A. S. (2014). Limites e potencialidades das rodas de conversa no cuidado em saúde: uma experiência com jovens no sertão pernambucano. Interface - Comunicação, Saúde, Educação, 18(2), 1299-1311.

Soto, R. V., Rubio, M. E. M., Star, M. J. V., Cárdenas, A. O., Preciado-Rangel, P., González, J. A., \& Esparza-Rivera, J. R. (2015). Cnidoscolus chayamansa hidropónica orgánica y su capacidade hipoglucemiante, calidad nutraceutica y toxicidad. Revista Mexicana de Ciências Agrícolas, 6(4), 815-825.

Van Der Horst, K., Brunner, T. A., \& Siegrist, M. (2011) Ready-meal consumption: Associations with weight status and cooking skills. Public Health Nutrition, 14(2), 239-245.

Como citar este artigo:

Peres, B. C. de A., Vidal, M. M. R., Gama, L. P., Pires, E. R., Reis, D. L., Silva, M. M., De Cnop, M. L., Pereira, A. F., \& da Silva, T. T. C. (2021). Oficina culinária como estratégia de articulação entre os movimentos sociais e a comunidade acadêmica para a promoção da alimentação saudável e sustentável: Relato de experiência. Revista Brasileira de Extensão Universitária, 12(2), 179-189. https://periodicos.uffs.edu.br/index.php/RBEU/article/view/11611/pdf 